\title{
Integrated Link Adaptation and Power Control to Improve Error and Throughput Performance in Broadband Wireless Packet Networks*
}

\author{
Kin K. Leung** \\ Li-Chun Wang \\ AT\&T Labs, Room A5-1D35 \\ 200 South Laurel Ave \\ Middletown, NJ 07748, U.S.A. \\ Phone: +1 732-420-9041 \\ Fax: +1 732-368-9426
}

Email: \{kkleung, lichun\}@ research.att.com

February 3, 2000

March 30, 2001 (Revised)

\begin{abstract}
In this paper, we prove that the problem of maximizing data throughput by adaptive modulation and power control while meeting packet error requirements is NP-complete. A heuristic algorithm for integrated link adaptation and power control is thus proposed to achieve specified error rates and to improve overall throughput for real-time applications in broadband wireless packet networks. The algorithm divides terminals into groups according to their signal path gains, and periodically adapts transmissions based on the required error rates, actual error statistics and average transmission power of each terminal group. Transmission power is adjusted by an enhanced Kalman-filter method to ensure successful reception. Extensive simulation results reveal that the algorithm consistently delivers the specified error performance, and attempts to maximize network throughput for a wide range of parameter settings.
\end{abstract}

* Parts of this paper were presented at IEEE VTC2000-Spring, Tokyo, Japan, May 2000 and IEEE WCNC, Chicago, IL, Sept. 2000.

** Please address correspondence pertinent to this submission to him. 


\section{INTRODUCTION}

As telecommuting and Internet access become increasingly popular, customers' demand for broadband network services has been growing significantly. In the very near future, broadband services are also expected to support real-time, multimedia services such as voice, image and video. Wireless access is one of the approaches to providing such services. In particular, the ETSI is in the process of establishing the protocol standards for the Enhanced Data rates for GSM Evolution (EDGE) system [SAEE98] as the third generation wireless network for high-speed services.

Using packet-switching technology, and multiple modulation and coding levels, the EDGE system employs a link-adaptation technique to adapt packet transmission to one of six coding levels [E98] (a current proposal has nine modulation and coding levels [E99]), where the highest data rate can exceed $550 \mathrm{Kbits} / \mathrm{sec}$. The main idea of link adaptation is to adapt the modulation and coding levels according to the channel and interference conditions in order to improve data throughput. For example, when the channel and interference conditions are poor, a low modulation level (i.e., few information bits per symbol) and/or heavy coding should be used in a packet transmission to enable correct signal detection. On the other hand, if the channel situations are favorable, high modulation level and/or light coding can be used to increase data rate.

Due to unreliable radio links, it is challenging to ensure quality of service (QoS) in terms of packet error rate (PER) in the wireless networks. For real-time services such as IP voice, music and video, stringent delay requirements severely limits or even precludes re-transmission of lost packets. Thus, tight delay requirements often translate into stringent requirements for PER. As a result, in order to support such real-time services, it is important to design the wireless networks such that the required QoS can be delivered to users.

Evidently, link adaptation is helpful in delivering QoS. Specifically, when the channel condition is poor, transmitters can lower modulation levels to decrease the requirements of signal-tointerference-plus-noise ratio (SINR) for correct signal detection. Lowering SINR requirements increases the probability of successful reception, thus helping to meet the PER objective. However, 
especially for interference-limited systems with sufficient traffic load, adapting even to the lowest modulation level may not always guarantee meeting the specified PER. In this case, increasing transmission power can improve signal strength and thus the SINR at the receivers. Hence, one can view power control as performing an active role in delivering the expected PER to users, while adaptive modulation plays a passive (or reactive) role. As discussed further below, the complementary roles of power control and link adaptation give us a very efficient approach to providing the required QoS to users. With this understanding, a key design problem for a wireless packet network like the EDGE system is: how to maximize the overall network throughput over the choice of modulation and coding levels, and transmission power, subject to meeting given PER requirements. In this paper, we propose and analyze an integrated algorithm of link adaptation and power control for the problem.

Our recent work [LW00] (and early results in [LW99]) on integrated power control and link adaptation represent our initial attempts in solving the problem. In particular, the proposed algorithm in [LW00] chooses the modulation and power level according to a power-stability criterion (to maintain stable transmission power in a long run). In this work, we find that the use of the stability criterion is actually unnecessary, when link adaptation and power control operation is based on the measured PER at the receivers and their requirements, provided that a feasible setting of modulation and power levels exists to support the required PER in the network under consideration. This is so because monitoring and controlling PER performance by the link adaptation and power control implicitly force transmission power to fall within a given dynamic range. Indeed, the algorithm proposed in this paper is based on monitoring the PER performance.

Let us briefly review other work on power control and link adaptation. Power control has been widely studied to combat interference in wireless networks; see e.g., [Z92], [RZ98] and [UY98]. Recently, [L99] proposes a Kalman-filter method for power control in packet-switched timedivision-multiple-access (TDMA) networks. An enhanced version of the Kalman-filter method is used to control transmission power here. Much work on link adaptation by adaptive modulation has 
been done for fading channels; see for example, [GC97] and [AG98]. For cellular systems, [QC99a] proposes iterative algorithms to maximize the overall network throughput by adaptive modulation and power control. Unfortunately, the formulation in [QC99a] does not consider the PER requirements (constraints). Evidently, it is difficult to extend the algorithms for real-time services with specific PER requirements. In [C99] and [QC99b], new adaptation schemes are proposed to improve overall data throughput for non-real-time data services. However, the use of link adaptation to deliver the specified PER for real-time, multimedia applications is an open issue, which is the topic to be addressed in this paper. Furthermore, we examine how power control, which is not considered in [C99] and [QC99b], is integrated with link adaptation for performance improvement.

The rest of this paper is organized as follows. We first present the operations and power control for the wireless packet networks under consideration in Section 2. In Section 3, we prove that the problem of maximizing network throughput while meeting the given PER requirements is NPcomplete. That is, no efficient algorithm for solving the problem exists. Then, a new algorithm for integrated link adaptation and power control based on monitoring PER performance is proposed in Section 4. In Section 5, we study the performance of the proposed algorithm by simulation. Finally, Section 6 presents our conclusion.

\section{NETWORK OPERATIONS AND POWER CONTROL}

Consider a packet-switched TDMA network with data rates up to several megabits per second, link lengths (or cell radius) typically less than 10 kilometers and operating frequency in the range of 1 to $5 \mathrm{GHz}$. Assume that each data message (e.g., an email) is divided into a number of packets, each of which can be transmitted in one time slot. Similar to other IP networks, when a transmitter (either a mobile terminal or a base station) sends a message, all its packets are transmitted in successive time slots. (For example, such corresponds to transmission in the same time slot of the consecutive TDMA frames [R96] in the GSM/EDGE system providing single time-slot service.) Similar to the EDGE system, the network under consideration allows transmitters to choose one of $M$ combinations 
of modulation and coding levels for transmission in each time slot, according to the link-adaptation algorithm in use. In this study, the key effect of using different modulation and coding levels is a change of SINR requirement for correct data reception. Thus, for brevity, we refer to the adaptation of modulation and coding levels simply as adaptive modulation here. Further, although the algorithm for integrated power control and adaptive modulation to be proposed below is applicable to both uplink (from terminal to base station) and downlink (from base station to terminal), our discussion will focus on the uplink transmission.

An enhanced Kalman-filter method is used to control transmission power. Using this method, each terminal continuously measures the interference power for its assigned radio channel, regardless of whether or not the base station associated with the terminal is transmitting data and whether or not the data being transmitted is intended for the terminal. The measurements are fed into a Kalman filter to predict future interference power. Let $\tilde{i}_{n}$ and $\tilde{I}_{n}$ denote the predicted interference power for slot $n$ in $\mathrm{mW}$ and $\mathrm{dBm}$, respectively. The time and measurement update equations for the Kalman filter for interference predictions are:

$$
\begin{gathered}
\tilde{I}_{n+1}=\hat{I}_{n} \\
\tilde{P}_{n+1}=\hat{P}_{n}+Q_{n} \\
K_{n}=\tilde{P}_{n}\left(\tilde{P}_{n}+R_{n}\right)^{-1} \\
\hat{I}_{n}=\tilde{I}_{n}+K_{n}\left(Z_{n}-\tilde{I}_{n}\right) \\
\hat{P}_{n+1}=\left(1-K_{n}\right) \tilde{P}_{n}
\end{gathered}
$$

where $\tilde{I}_{n}$ and $\hat{I}_{n}$ are the a priori and a posteriori estimate of interference power in $\mathrm{dBm}$ for slot $n, \tilde{P}_{n}$ and $\hat{P}_{n}$ are the a priori and a posteriori estimate-error variance, $K_{n}$ is the Kalman gain, $Z_{n}$ is the measured interference power (i.e., the actual interference plus measurement error), and $Q_{n}$ and $R_{n}$ are the variance for the process noise (i.e., the change of interference power of one slot relative to the 
previous one) and the interference measurement noise (or error), respectively. Based on measurements up to slot $n, \tilde{I}_{n+1}$ in (1) gives the predicted interference power for slot $n+1$.

Let $\bar{Z}_{n}$ be the average interference measurements over the last $W$ frames prior to slot $n$. Hence, we have

$$
\bar{Z}_{n}=\frac{1}{W} \sum_{i=n-W+1}^{n} Z_{i}
$$

We can estimate $Q_{n}$ by

$$
Q_{n} \approx \frac{1}{W-1} \sum_{i=n-W+1}^{n}\left(Z_{i}-\bar{Z}_{n}\right)^{2}
$$

We note that $Q_{n}$ in (7) is an estimate of the sum of the variances for the interference power and measurement noise because the measurement $Z_{i}$ in (6) is the sum of the actual interference power and measurement error. However, since the fluctuation of the interference power can reach as much as tens of decibels, which is much higher than typical measurement errors, (7) represents a good estimate for $Q_{n}$.

It is also worth noting that $R_{n}$ depends on the actual error characteristics of interference measurements. To illustrate our ideas without considering details of the measurement error characteristics, we assume for simplicity in this paper that $R_{n}$ is given by

$$
R_{n}=\eta Q_{n}
$$

where $\eta$ is a given constant between 0 and 1 . This range is appropriate because as mentioned above, $Q_{n}$ in (7) includes the measurement-error variance. For a given $\eta$, we effectively consider the corresponding value for the variance of measurement errors.

The predicted interference $\tilde{i}_{n}$ in (1) in mW can be used to control transmission power $p_{n}$ for slot $n$ : 


$$
p_{n}=\min \left\lceil\frac{\gamma^{*} \tilde{i}_{n} \delta_{n}}{g_{n}}, p_{\max }\right\rfloor
$$

where $\gamma^{*}$ is the SINR target for the chosen modulation level, $\tilde{i}_{n}$ is the interference-plus-noise power $(\mathrm{mW})$ in slot $n$ predicted by the Kalman filter, $\delta_{n}$ is an error margin (to be discussed below), and $g_{n}$ is the (estimated) path gain between the terminal that transmits in slot $n$ and its base station.

The error margin $\delta_{n}$ is obtained by tracking the accuracy of the interference power predicted by (1). More precisely, let $\Delta$ (a random variable in $\mathrm{dB}$ ) be the error of the Kalman-filter prediction and the error for slot $n$ be

$$
E_{n}=Z_{n}-\tilde{I}_{n}
$$

where $Z_{n}$ and $\tilde{I}_{n}$ are the measured and predicted interference power in $\mathrm{dBm}$ for slot $n$, respectively. Based on the $E_{n}$ 's, we approximate the cumulative probability function (CDF) for $\Delta$. Towards this end, let there be $J$ intervals of prediction error and the range of the $j^{\text {th }}$ interval be $\left(a_{j}, a_{j+1}\right]$, as shown in Figure 1. To cover the full range of possible errors, one may need to set $a_{1}=-\infty$ and $a_{J+1}=\infty$. For each time slot $n>0$ and each $j=1$ to $J$, compute the following

$$
P_{n}^{j}= \begin{cases}\phi P_{n-1}^{j} & \text { if } E_{n}>a_{j+1} \\ \phi P_{n-1}^{j}+1-\phi & \text { otherwise }\end{cases}
$$

where $P_{n}^{j}$ is the approximate probability of $\Delta \leq a_{j+1}$ based on the error sequence $E_{n}$ up to slot $n$ with $P_{0}^{j}=1$ for all $j=1$ to $J$ initially, and $\phi$ is a properly chosen parameter between 0 and 1 .

To provide a physical interpretation for (11), let us assume $\phi=1-1 / m$ where $m$ is a positive integer. Without loss of generality, we consider the probability for $\Delta \leq a_{2}$ (i.e., the probability mass associated with the first error interval) based on prediction errors $E_{n}$ 's in a sliding window of last $m$ slots. Furthermore, suppose that $E_{n} \leq a_{2}$ for all $n=1$ to $m$. As a result, (11) yields $P_{m}^{1}=1$, since $P_{0}^{1}=1$ initially. Now, $E_{m+1}$ becomes available for slot $m+1$. If $E_{m+1}>a_{2}$, then $P_{m+1}^{1}=(1-1 / m)$ 
according to (11). The interpretation of this is that among the $E_{n}$ for $n=2$ to $m+1$, only $E_{m+1}$ is greater than $a_{2}$. So, by definition of $P_{n}^{j}, P_{m+1}^{1}=(m-1) / m$, which is identical to that by (11). On the other hand, if $E_{m+1} \leq a_{2}$, (11) yields $P_{m+1}^{1}=1$, which is also consistent with the probability definition. In essence, given that the prediction errors become available one at a time, (11) uses an exponential-smoothing technique to handle prediction errors over a sliding window of time slots and to approximate the CDF for $\Delta$.

Let $\Delta_{n}$ be a specified $\omega^{\text {th }}$ percentile (e.g., for 90th percentile, $\omega=0.9$ ) of $\Delta$ based on the error statistics up to slot $n$. We approximate $\Delta_{n} \approx a_{k+1}$ where $k$ is the smallest from 1 to $J-1$ such that $P_{n}^{k} \geq \omega$. For the example in Figure 1, the 90th percentile for $\Delta$ based on statistics up to slot $n$ is approximated by $a_{j+2}$ as $P_{n}^{j}<0.9$ and $P_{n}^{j+1} \geq 0.9$. Let $\delta_{n}$ and $\tilde{i}_{n}$ be the linear-scale equivalent of $\Delta_{n}$ and $\tilde{I}_{n}$, respectively. The corresponding percentile of the interference power in $\mathrm{mW}$ is the product of $\tilde{i}_{n}$, predicted by the Kalman filter in (1), and $\delta_{n}$. Accordingly, the transmission power for slot $n$ is determined by (9).

We note that $\delta_{n}$ actually represents an error margin, which depends on the accuracy of the interference prediction by the Kalman filter and the specified confidence probability $\omega$. Nevertheless, the error margin is chosen dynamically and appropriately with a goal of delivering the SINR target $\gamma^{*}$ regardless of the actual message length and control delay (see [L99] and [L00]). It is also worth noting that the power control requires periodic exchange of control information between the receiver and the transmitter. Readers are referred to [CLQT00] for a discussion of such exchange.

\section{PROBLEM COMPLEXITY}

In this section, we prove that a simple form of the problem of maximizing network throughput subject to meeting a given PER requirement posed in Section 1 is NP-complete [GJ79]. More precisely, let the system have $N$ terminals in $N$ different co-channel sectors concurrently transmitting packets to their base stations (i.e., having $N$ radio links) at each time slot. The modulation level and 
power for link $i$ at a given slot are denoted by $L(i)$ and $p(i)$, respectively. Let there be $M$ modulation levels, indexed by 1 to $M$, and $K$ discrete levels of transmission power, denoted by real numbers $t_{i}$ for $i=1$ to $K$. As a result, for each link $i, L(i) \in\{1,2, \ldots, M\}$ and $p(i) \in\left\{t_{1}, t_{2}, \ldots, t_{K}\right\}$. In general, one can represent the data rate and the required SINR detection threshold associated with each modulation level as nonlinear functions of the level index. However, we show below that even if the functions are linear, the optimization problem is already difficult to solve. Specifically, the data rate and the detection threshold for link $i$ are assumed to be $\alpha L(i)$ and $\beta L(i)$, respectively, where $\alpha$ and $\beta$ are proportionality constants. Let $\mathbf{L}=(L(1), L(2), \ldots, L(N))$ and $\mathbf{p}=(p(1), p(2), \ldots, p(N))$. Using this notation, the problem of maximizing the overall throughput $\lambda$ for each time slot is:

$$
\max _{\mathbf{L}, \mathbf{P}} \lambda=\sum_{i=1}^{N} \alpha L(i)
$$

subject to $\frac{g_{i i} p(i)}{\sum_{j \neq i} g_{i j} p(j)+\sigma} \geq \beta L(i)$ for all $i=1$ to $N$

where $g_{i j}$ is the path gain from the transmitter of link $j$ to the receiver of link $i$ and $\sigma$ is the receiver noise power. We note that the above SINR constraints in (13) are used to represent the PER requirement for the chosen modulation level. Further, the throughput is maximized for each time slot. For the packet-switching networks under consideration, the values of $g_{i j}$ 's in (13), and thus the optimal $\mathbf{L}$ and $\mathbf{p}$ change from one time slot to another. However, the optimization in (12) is also valid for circuit-switching wireless networks where these quantities are independent of time.

Theorem 1. The problem of maximizing the overall throughput in (12) subject to the SINR (or equivalently PER) requirement in (13) is NP-complete.

Proof. The optimization problem (12) is nonlinear due to the products of $p(j)$ 's and $L(i)$ 's in (13). Let $Q_{i j}=L(i) p(j)$ for all $i, j=1$ to $N$. Thus, $Q_{i j} \in\left\{t_{1}, \ldots, t_{K}, 2 t_{1}, \ldots, 2 t_{K}, \ldots, M t_{1}, \ldots, M t_{K}\right\}$. Since $Q_{i j}$ 's are finite, real numbers, we can express each $Q_{i j}$ in terms of binary (zero or one) variables 
$X_{i j k}$ 's as $Q_{i j}=\sum_{k=a_{i j}}^{\bar{a}_{i j}} X_{i j k} 2^{k}$ where $a_{i j}$ and $\bar{a}_{i j}$ are properly chosen integers according to the accuracy tolerance of the binary representation. Similarly, we express $L(i)=\sum_{k=b_{i}}^{\bar{b}_{i}} Y_{i k} 2^{k}$ and $p(i)=\sum_{k=c_{i}}^{\bar{c}_{i}} Z_{i k} 2^{k}$. Consequently, (12) becomes a nonlinear zero-one integer programming problem. Now, we apply the technique in [C69] to linearize the problem by introducing new variables to replace the cross-product terms. Then, the proof is completed by using the fact that the resultant, linear integer programming is NP-complete [GJ79, p.245].

As the optimization problem is NP-complete, it is unlikely that any efficient algorithm may exist. Thus, we devise a heuristic approach in the following with a primary goal of delivering the specified PER, while attempting to maximize the network throughput as a secondary objective.

\section{INTEGRATED LINK ADAPTATION AND POWER CONTROL}

There are two key factors for efficient link-adaptation schemes. First, to maximize the network throughput, it is desirable to have a link-adaptation technique that adapts quickly to changes of radio conditions. On the other hand, to guarantee the required PER, it is advantageous to adapt the link according to the actual error performance (where packet error can be identified by use of cyclicredundancy code). Since error statistics require a long time to accumulate, especially for meeting stringent PER requirements, link adaptation based on per-user error performance will be too slow in responding to changes of channel conditions. As a compromise, we propose to divide all terminals in each co-channel sector into groups and to perform link adaptation on a per-terminal-group basis according to the error performance of each group. This way, by monitoring the error performance of all traffic associated with a terminal group, the statistics collection time can be shortened significantly, thus enabling quick link adaptation to improve data throughput while meeting the error requirements. 


\subsection{Terminal Grouping by Signal Path Gains}

The quality of a radio link between a terminal and its associated base station can typically be characterized by three parameters: the signal path gain (including shadow fading), and signal transmission and interference power. In the packet networks under consideration, both transmission and interference power change constantly. In our view, signal path gain is the most intrinsic parameter for link quality. So, we propose to use the signal path gain as a criterion for the terminal grouping. Terminals of the same group are expected to have similar link quality, and cause similar amount of interference to others.

Another supporting reason for using signal path gain as the grouping criterion is that signal and interference path gains are almost uncorrelated. To illustrate our idea, consider the cellular layout and channel assignment of frequency reuse factor of 2 in Figure 2 [WL00], where 2,000 terminals are randomly populated at fixed locations in each cell. With typical radio propagation assumptions (see Section 5 for details), Figure 3 shows the relationship between the signal path gain (shadowing included) from a terminal to its associated base station and the sum of path gains from the terminal to all other co-channel base stations, which receive interference from the terminal for uplink transmission. The sum of interfering path gains reflects the potential impacts of interference caused by the terminal. It is found that the signal path gain and the sum of interfering path gains have a very small correlation coefficient of 0.018 . Such uncorrelated relationship is desirable for our purposes. Otherwise, if it were a strong positive correlation, the link adaptation based on such a terminal group basis would make unstable changes of modulation level.

One way to perform the terminal grouping is to determine the range of the signal path gain for a given network. Then, the range is divided into several (not necessarily uniform) regions, according to a given set of region parameters. Terminals with corresponding signal path gain in each region form a group. To speed up the collection of error statistics uniformly for various terminal groups, it is desirable to have a roughly equal number of terminals in each group, based on an assumption that 
each terminal has similar traffic load characteristics. As a natural candidate, the number of terminal groups can be chosen to be equal to the number of modulation levels in the system such that each group may be transmitting or receiving at a distinct modulation level. Of course, the number of groups should be selected based on the number of active terminals; too few terminals per group will defeat the purpose of terminal grouping in terms of shortening the statistics collection time.

It is worth noting that depending on mobility, a given terminal may belong to different terminal groups in a dynamic way. Each terminal periodically estimates its signal path gain (e.g., by use of the control channel for handoff purposes in GSM system [R96]). One approach to reducing unnecessary group re-assignment is to feed the periodic estimates of signal path gain for each terminal into a lowpass filter. Then, comparing the "smoothed" signal-path gain against the region parameters determines the affiliated terminal group for the terminal. Based on this method, unless a terminal is extremely mobile, the smoothed quantity provides a reasonable representation of the signal-path gain.

\subsection{Adaptation Algorithm by Terminal Grouping}

The new algorithm for link adaptation and power control for uplink transmissions is outlined below (while similar operations apply to downlink):

1. For each co-channel sector, its base station continuously collects the error statistics, and computes the PER $P_{E}$ for every $K$ packet transmissions from terminals of each group. In addition, it keeps track of the average transmission power $\bar{p}$ of the $K$ packet transmissions, regardless of their modulation levels, for each terminal group.

2. This step is taken every time after $K$ packets have been transmitted by terminals of the same group. If $P_{E}$ is higher than the required value $P_{R}$, adjust the modulation level down by one level for the next $K$ packet transmissions by terminals in the group. The purpose of stepping down the modulation level is to lower the required SINR for correct reception, thus improving the PER when needed. Further, if $\bar{p}$ is less than a given threshold $p_{t}$ (e.g., $15 \mathrm{dBm}$ ), step up the 
modulation level by one level for the next $K$ transmissions from the terminal group. The idea there is to utilize unused power to increase data rate, if possible.

3. To achieve the PER requirement, the enhanced Kalman-filter power control in Section 2 is used to adjust the transmission power for each time slot according to (9) to achieve the SINR target for the adapted modulation level.

4. Due to different fading conditions for various terminals, using the same $\gamma^{*}$ for a given modulation level in (9) may not necessarily guarantee the required PER performance for each terminal in the same group. To ensure that, each terminal is allowed to use slightly different values of $\gamma^{*}$ as follows. Each modulation level has a nominal SINR target $\gamma$ and each terminal maintains an adjustment parameter, denoted by $\Theta$ and $\theta$ in $\mathrm{dB}$ and linear scale, respectively. Initially, each terminal sets $\Theta=0$ and $\theta=1$. Each terminal monitors its PER for every $K$ packets transmitted by the terminal. If the updated PER fails to meet the required $P_{R}, \Theta$ is increased by a pre-specified, fixed quantity (e.g., $0.5 \mathrm{~dB}$ ). Otherwise, $\Theta$ is decreased by the same quantity, unless $\Theta$ is 0 . Then, for the next $K$ packet transmissions by the terminal, the linear-scale equivalent $\theta$ is used to obtain the SINR target for the chosen modulation level by substituting $\gamma^{*}=\gamma \theta$ into (9) for power control. (The same $\theta$ is applicable to determining $\gamma^{*}$ for any modulation level chosen for the terminal.) The key idea here is to slightly increase the SINR target by $\Theta \mathrm{dB}$ for achieving the required error performance for individual terminals.

The choice of $K$ in Step 1 should be large enough to ensure statistical significance of the measured error rate relatively to a given PER requirement $P_{R}$. A reasonable choice is to set $K$ to be 10 or 20 times of $1 / P_{R}$. For example, when $P_{R}=0.02$, choose $K=500$ or 1,000 to ensure the accuracy of the measured PER performance.

It is worth noting that Step 2 steps down the modulation level due to unsatisfactory PER performance, and moves it up in case of under-utilized power. In special cases, it is possible that the PER does not meet the required performance and the average transmission power is also below the 
threshold $p_{t}$. We expect that such is probably due to the fact that the Kalman filter cannot predict interference-plus-noise power accurately enough. Since the power control in (9) includes the error margin $\delta_{n}$, which possibly changes from one time to the next, slight increase in $\delta_{n}$ because of the inaccurate predictions (thus using a small fraction of unused power) may be enough to meet the PER requirement. Thus, the modulation level remains unchanged in Step 2 for those cases, with a hope that the PER becomes satisfactory for the next $K$ packet transmissions by power control.

We also remark that the dynamic adjustment for $\gamma^{*}$ according to the measured and required packet error rate in Step 4 is similar to the outer-loop power control for CDMA systems [LM98].

\section{PERFORMANCE STUDY}

\subsection{Simulation Model}

We use computer simulation to study the performance of the proposed algorithm for link adaptation and power control based on the terminal grouping, which is referred to as the terminalgrouping method below. The cell layout and interleaved channel assignment (ICA) with a frequency reuse factor of 2 [WL00] in Figure 2 is simulated. In this ICA scheme, there are 4 sets of channels and sectors with the same label in the figure share the same channel set. Each cell is divided into 4 sectors, each of which is served by a base station antenna at the center of the cell. The beamwidth of each base station antenna is $60^{\circ}$, while terminals have omni-directional antennas. Each radio link between a terminal and its base station is characterized by a path-loss model with an exponent of 4 and lognormal shadow fading with a standard deviation of $8 \mathrm{~dB}$. Fast fading is not considered in this study. Cell radius is assumed to be $1 \mathrm{Km}$ and the path loss at $100 \mathrm{~m}$ from the cell center is $-70 \mathrm{~dB}$. Thermal noise power at the receiver is fixed and equal to $-110 \mathrm{dBm}$. Each sector is populated with 500 terminals randomly and each of them selects the base station that provides the strongest signal

power. Terminals are assumed to remain stationary throughout the simulation. For convenience, terminals in all cells are assumed to be synchronized at the slot boundary for transmission. 
Furthermore, unless stated otherwise, we assume $100 \%$ traffic load in this study; that is, there are always terminals ready for transmission in each co-channel sector. Message length is assumed to be Pareto distributed [L99] with an average of 10 packets here, except for Figure 5 where each message consists of 5 packets on average.

The enhanced Kalman-filter method is used to control transmission power for each time slot in each co-channel sector in Figure 2. For tracking the CDF of the prediction error $\Delta, \phi$ in (11) is set to be 0.999 (approximately equal to tracking the error over a sliding window of 1,000 slots) and the number of error intervals $J$ is 100. For a given PER requirement $P_{R}$, the $\omega^{\text {th }}$ percentile of the prediction error with $\omega=1-P_{R}$ is used in determining the error margin $\delta_{n}$ for adjusting power in (9). For example, when $P_{R}=0.02, \delta_{n}$ is the $98^{\text {th }}$ percentile of the prediction error. In any event, transmission power is limited between 0 to $30 \mathrm{dBm}$. The average power threshold, $p_{t}$, for determining stepping up the modulation level in Step 2 of the algorithm is $15 \mathrm{dBm}$. As illustrative examples, $W$ and $\eta$ in (6) and (8) are set to be 30 and 0.5 , respectively. The model also assumes that interference power in one time slot can be measured and used to determine the power for the next slot.

We assume that the system has 6 modulation levels. The SINR detection requirements and the corresponding data throughput for each modulation level are given in Table 1. For example, for a packet transmission using modulation level 1, if the SINR at the receiver is greater than $10 \mathrm{~dB}$, the packet is received successfully and the associated data throughput is $22.8 \mathrm{Kbps}$. Naturally, the SINR requirements are also used as the targets $\gamma^{*}$ for various modulation levels to control power in (1). For simplicity, slow adjustment of SINR targets for individual terminals in Step 4 of the algorithm is not considered in the simulation. Parameters in Table 1 are actually adapted from Table 3 and estimated from Figure 20 of [E98]. With 6 modulation levels, all 500 terminals in each sector are divided into 6 groups of equal size according to their signal path gain. Initially, the group with the weakest to the strongest signal path gain uses modulation level 1 to 6 for transmission, respectively. Then, according to the algorithm, the modulation level is re-adapted every $K=1,000$ packets transmitted by each terminal group. For each parameter setting, the simulation model was run for 0.4 million time 
slots and performance results presented below were obtained for the middle cell in Figure 2.

\subsection{Performance Results and Discussions}

To set up a basis for comparison, we consider a simple link-adaptation scheme without power control (PC) that chooses the modulation level according to the SINR measurement of the previous time slot. Specifically, the scheme compares the SINR measurement with the detection requirements in Table 1. For example, when the measurement lies between 12 and $16 \mathrm{~dB}$, modulation level 2 is used for transmission in the next time slot. Every sector makes such selection for its transmitting terminal independently. This SINR scheme is referred to as Case A in Table 2. Cases B to D are identical to Case A, except that the enhanced Kalman method is now used to control transmission power according to the various PER requirements $P_{R}$. To help us understand the algorithm characteristics, Cases E to G correspond to the link-adaptation method by terminal grouping without power control, while Cases $\mathrm{H}$ to $\mathrm{J}$ represent our proposed algorithm. Table 2 presents the throughput, the PER for packets transmitted at different modulation levels, and the overall PER averaged over all levels for these cases.

First of all, we observe from the table that when power control is not used, Cases A and E to G for both the SINR and terminal-grouping methods cannot control the PER effectively. For the SINR method, it is so because the previous SINR measurements may not accurately predict SINR performance in future time slots because of the burstiness of packet transmission in the wireless packet environment. As a result, the chosen modulation level may not lead to successful packet reception as the radio conditions can change drastically in time. As for Cases $\mathrm{E}$ to $\mathrm{G}$, the terminalgrouping method adapts the modulation level according to the requirement $P_{R}$. However, the scheme is not effective in delivering the PER performance because the link adaptation takes place only periodically and the radio conditions can vary significantly from one time slot to another during the adaptation period. 
As the results for Cases B to D show, the SINR scheme with the enhanced Kalman power control is still not quite effective in achieving the specified error performance because SINR measurements may not accurately reflect future link quality. In contrast, according to the specified PER requirement $P_{R}$, the proposed algorithm adapts transmissions at appropriate modulation levels, and adjusts transmission power to meet the SINR detection threshold. Consequently, as shown in Table 2, the PER performance for Cases $\mathrm{H}$ to $\mathrm{J}$ generally comes very close to the PER target $P_{R}$. One can observe that the PER for transmission at modulation level 1 in Case $\mathrm{H}$ is noticeably higher than the required 2\% PER. This reveals that the radio conditions may not support the very stringent PER performance for a very small fraction of terminals. In any event, the overall PER of $2.7 \%$ is very close to the target of $2 \%$ in that case. As intuitively expected, when $P_{R}$ becomes stringent, the throughput is reduced in Cases $\mathrm{H}$ to $\mathrm{J}$.

We also compare the performance of the terminal-grouping method with that reported in [LW99]. The latter algorithm chooses modulation level and transmission power according to a power-stability criterion to maintain stable transmission power in a long run. As mentioned earlier, such use of the stability criterion is unnecessary as the new algorithm monitors and operates based on the measured PER. To show a comparison between their performance, we include results for Method $\mathrm{D}$ in Table 2 of [LW99] as Case K in Table 2 here. Despite differences in the link-adaptation algorithms, the error performance for Case $\mathrm{K}$ is very similar to that of Case H. However, our new algorithm yields about 10\% more throughput than the one in [LW99]. Furthermore, our extensive testing shows that the new algorithm is more robust in delivering desirable performance for a wide range of settings than the previous one.

It is important to point out that the ability to control the PER to meet the specified targets by the proposed algorithm comes at a price of reduced throughput. In fact, this represents an interesting tradeoff between maximizing throughput and controlling PER performance. For example, one can see from Table 2 that the SINR method in Cases A to D yields almost 1/3 more throughput than Cases $\mathrm{H}$ to $\mathrm{J}$ of the terminal-grouping method. Nevertheless, to meet the PER requirements for 
specific applications, network designers can use the integrated algorithm for power control and link adaptation to achieve a desirable tradeoff between the PER and throughput.

Table 3 presents the probability distribution of the modulation levels for actual packet transmission for the proposed algorithm for various target PER. For example, for terminal group 4, the probability of packet transmission using modulation level 4 is $0.178,0.338$ and 0.454 for the target PER of 5\%,10\% and 15\%, respectively. This confirms the expected operations of the proposed algorithm because for less stringent PER requirement, the algorithm will tend to step up the modulation level for transmission.

Finally, we study the packet error and throughput performance of the proposed algorithm with partial traffic loading. For a given loading, after a terminal completes a message transmission, its associated sector remains idle for a random number of time slots before a next terminal in the sector is allowed to start a new message transmission. The duration of an idle period is geometrically distributed and its average is determined according to the average message length and the traffic loading. Table 4 presents the measured PER of the proposed algorithm for various target (required) PER, traffic load conditions and average of 10 and 5 packets per message (denoted by $L=10$ and 5). We observe that the proposed algorithm indeed can deliver the required PER performance as the measured PER comes very close to the target values in all cases considered.

Figures 4 and 5 show the corresponding throughput performance as a function of target PER for $L=10$ and 5 , respectively. It is interesting to note for both figures that the throughput generally increases as traffic load decreases. This is a very desirable feature because the power control in the proposed algorithm will detect reduced interference power when traffic load decreases, thus lowering the transmission power needed to yield the SINR target $\gamma^{*}$ in (9). In turn, comparing the average transmission power with a fixed threshold $p_{t}$ by Step 2 of the algorithm in Section 4.2 will likely step up the modulation level for transmission, which results into increased data throughput. 
Second, Figures 4 and 5 reveal that for a given traffic load, the throughput does not always improve as the target PER is increased (relaxed). Evidently, for very stringent PER requirement, the algorithm will force most of the transmissions using the more robust modulation, which yields a low throughput. On the other hand, when the required PER is too high, the algorithm tends to allow too many packets transmitted using high modulation levels, which result into unsuccessful reception and lowered throughput. For a given traffic load and message length characteristics, there appears to be an "optimal" target PER that can maximize the network throughput. Lastly, by comparing Figures 4 and 5, it is found that for a given traffic load and target PER, the throughput for $L=10$ tends to be slightly higher than that for $L=5$. This is so because when $L=10$, the interference temporal correlation is stronger than that for $L=5$. As a result, the Kalman filter predicts interference power more accurately, thus reducing the error margin $\delta_{n}$ in (9). In turn, lowered transmission power and interference enable the system to adapt high modulation levels for transmission for the throughput improvement.

\section{CONCLUSIONS}

The problem of maximizing data throughput by adaptive modulation/coding and power control while meeting requirements of packet error rate has been shown to be NP-complete. A heuristic algorithm for integrated link adaptation and power control has been proposed to achieve specified error rates while improving overall throughput for real-time applications in broadband wireless networks. The algorithm divides terminals into groups according to their signal path gains, and adapts the transmissions based on the required error rates, actual error statistics and the average transmission power of each terminal group. Transmission power is adjusted by an enhanced Kalmanfilter method to ensure correct reception.

The performance of the proposed algorithm has been studied by computer simulation. Our results reveals that the algorithm delivers the required error performance, and attempts to improve network throughput at the same time. It can take advantage of reduced interference for decreased 
traffic load to further improve throughput by transmitting at high modulation levels. Extensive testing shows that the algorithm is very robust and efficient. By having various packet error requirements, the algorithm is applicable to real-time, multi-media services. The new algorithm can also serve as a useful tool for achieving a desirable tradeoff among throughput, packet error rate and coverage in the networks.

Acknowledgment. Thanks are due to Justin Chuang for his information on the EDGE system, discussion and comments. The authors also thank Larry Greenstein, Paul Henry and Timiri Shailender for their comments.

\section{REFERENCES}

[AG98] M.-S. Alouini and A.J. Goldsmith, "Adaptive Modulation over Nakagami Fading Channels," preprint, 1998.

[C69] W.W. Chu, "Optimal File Allocation in a Multiple Computer System," IEEE Trans. on Computers, C-18, No.10, pp.885-889, Oct. 1969.

[C99] J.C. Chuang, "Improvement of Data Throughput in Wireless Packet Systems with Link Adaptation and Efficient Frequency Reuse," Proc. IEEE Veh. Tech. Conf., Houston, TX, May 1999, pp.821-825.

[CLQT00] J. Chuang, K.K. Leung, X. Qiu, S. Timiri and L.-C. Wang, "Power Control for Wireless Packet Voice with Application to EDGE System," Proc. of IEEE Globecom 2000, San Francisco, CA, Nov. 2000, pp. 213-219.

[E98] "EDGE - Evaluation of 8-PSK," ETSI SMG2, WPB\#4, Finland, April 20-24, 1998, Source: Ericsson. 
[E99] "EDGE: Concept Proposal for Enhanced GPRS," ETSI SMG2, WPA/WPB, Tucson, Arizona, May 31 to June 4, 1999, Source: Ericsson.

[GC97] A.J. Goldsmith and S.-G. Chua, "Variable-Rate Variable-Power MQAM for Fading Channels," IEEE Trans. on Commun., Vol. 45, No. 10, Oct. 1997, pp.1218-1230.

[GJ79] M.R. Garey and D.S. Johnson, Computers and Intractability: A Guide to the Theory of NPCompleteness, W.H. Freeman and Company, San Francisco, 1979.

[L99] K.K. Leung, "A Kalman-Filter Method for Power Control in Broadband Wireless Networks," Proc. IEEE INFOCOM'99, New York, NY, March 1999, pp.948-956.

[L00] K.K. Leung, "Power Control by Kalman Filter with Error Margin for Wireless IP Networks," Proc. of IEEE WCNC’00, Chicago, IL, Sept. 2000, pp. 980-985.

[LM98] J. S. Lee and L. E. Miller, CDMA Systems Engineering Handbook, Artech House (1998).

[LW99] K.K. Leung and L.C. Wang, "Integrated Power Control and Link Adaptation for QoS in Broadband Wireless Networks," Proc. IEEE PIMRC'99, Osaka, Japan, Sept. 1999, pp.11741180.

[LW00] K.K. Leung and L.C. Wang, "Controlling QoS by Integrated Power Control and Link Adaptation in Broadband Wireless Networks," European Trans. on Telecommunications, Vol. 11, No. 4, July/August 2000, pp. 383-394.

[QC99a] X. Qiu and K. Chawla, "On the Performance of Adaptive Modulation in Cellular Systems," IEEE Trans. on Commun., Vol. 47, No. 6, June 1999, pp. 884-895.

[QC99b] X. Qiu and J.C. Chuang, "Link Adaptation in Wireless Data Networks for Throughput Maximization Under Retransmissions," Proc. IEEE ICC, Vancouver, Canada, June 1999, pp.1272-1277. 
[R96] T. S. Rappaport, Wireless Communications: Principles and Practice, New York: IEEE Press and Prentice Hall, 1996.

[RZ98] Special issue on power control, Wireless Networks 4 (1998) 3, Z. Rosberg and J. Zander (Ed.).

[SAEE98] P. Schramm, H. Andreasson, C. Edholm, N. Edvardssona, M. Hook, S. Javerbring, F. Muller and J. Sköld, "Radio Interface Performance of EDGE, a Proposal for Enhanced Data Rates in Existing Digital Cellular Systems," Proc. of IEEE VTC, Ottawa, Canada, May 1998, pp.1064-1068.

[UY98] S. Ulukus and R.D. Yates, "Stochastic Power Control for Cellular Radio Systems," IEEE Trans. on Commun., Vol. 46, June 1998, pp.784-798.

[WL00] L.C. Wang and K.K. Leung, "A High-Capacity Wireless Network by Quad-Sector Cell and Interleaved Channel Assignment," IEEE Journal on Selected Areas in Communications, Vol. 18, No. 3, March 2000, pp. 472-480.

[Z92] J. Zander, "Performance of Optimum Transmitter Power Control in Cellular Radio Systems," IEEE Trans. on Veh. Tech., Vol. 41, No. 1, Feb. 1992, pp.57-62. 
Fig.1. Approximate CDF for Interference Prediction Error

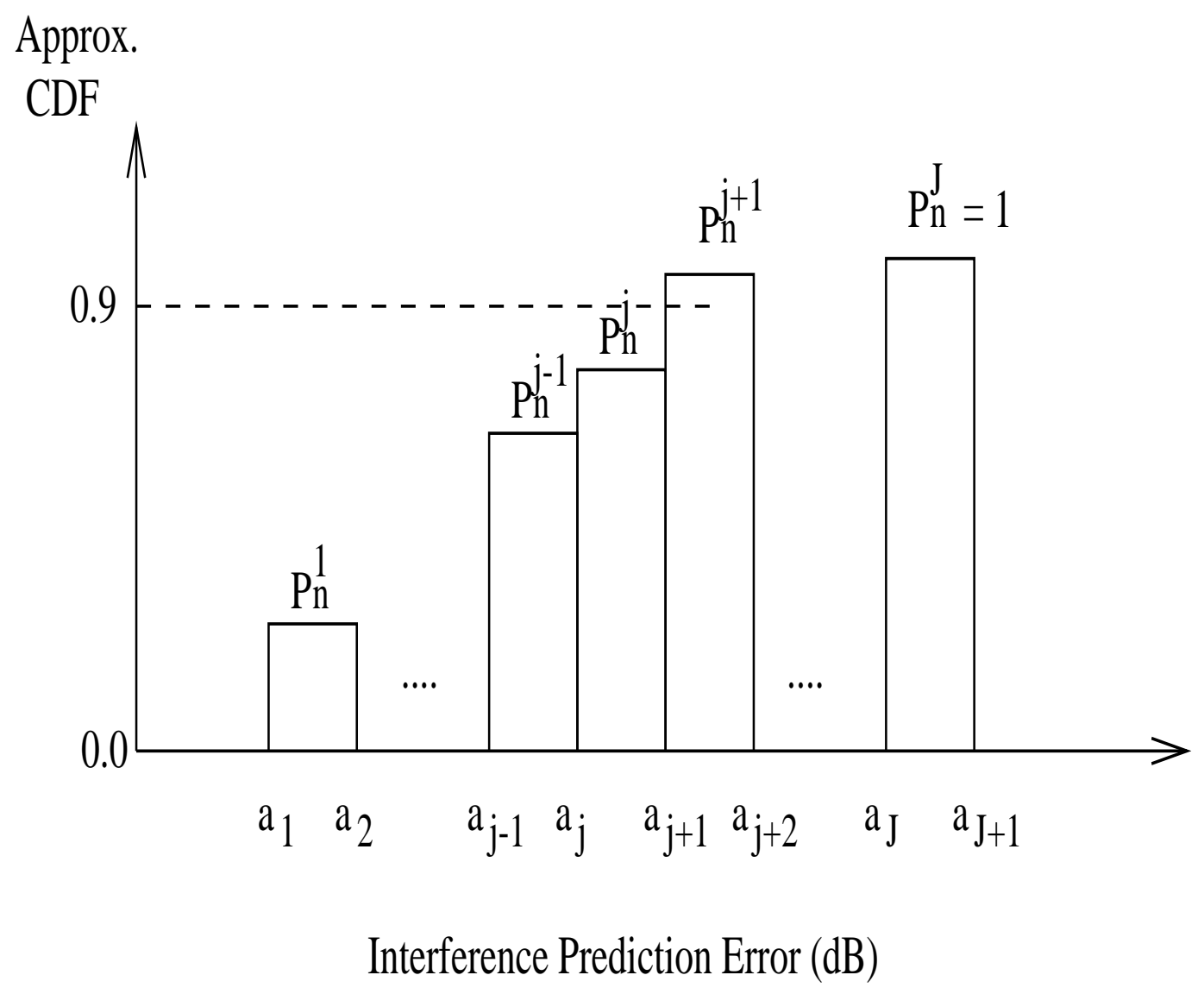


Fig.2. A 4-Sector Cell Layout and ICA

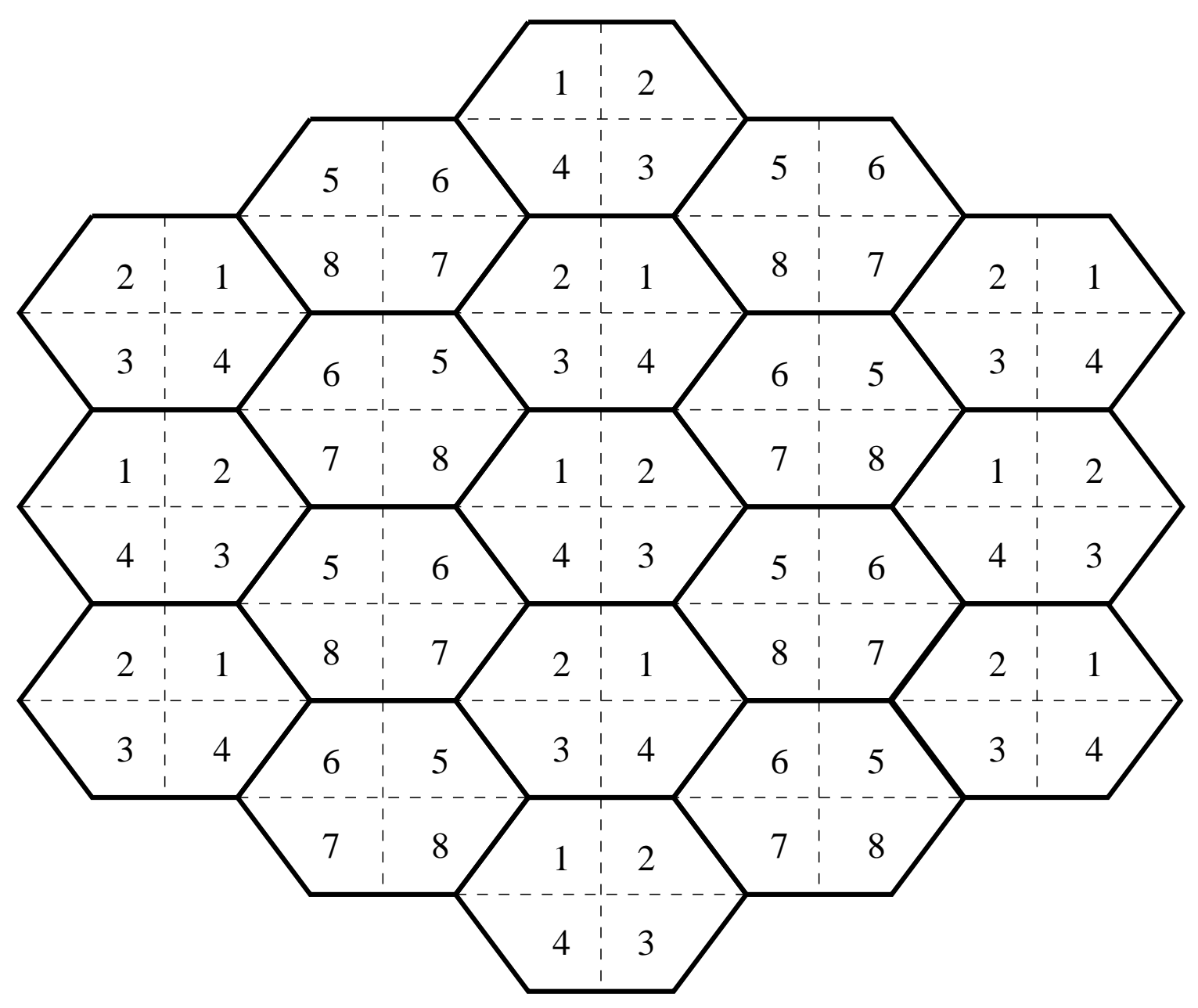


Fig.3. Interference Vs. Signal Path Gain

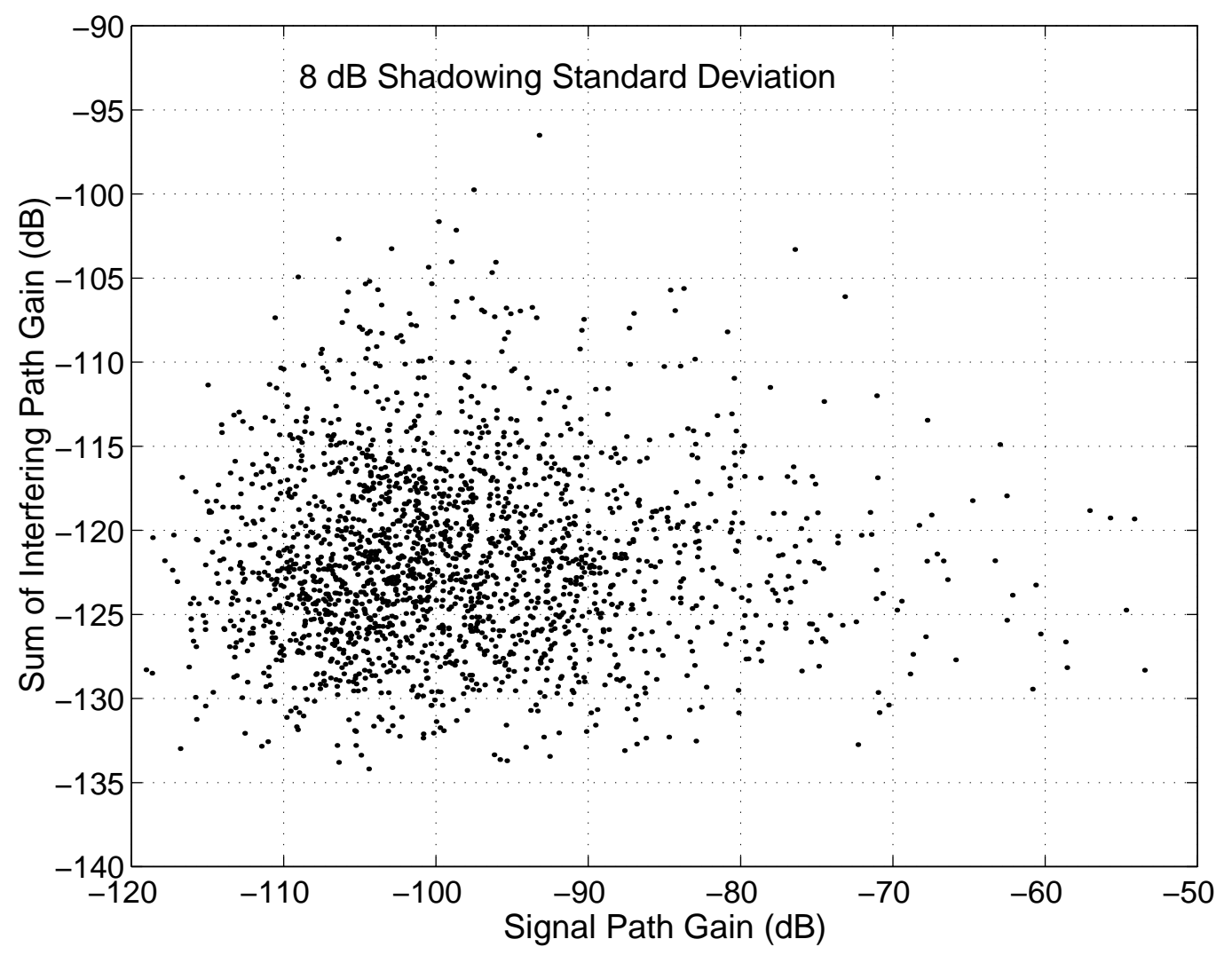


Table 1. SINR Requirement \& Throughput.

\begin{tabular}{|c|c|c|}
\hline $\begin{array}{c}\text { Modulation } \\
\text { Level }\end{array}$ & $\begin{array}{c}\text { SINR Detection } \\
\text { Requirement (dB) }\end{array}$ & $\begin{array}{c}\text { Throughput } \\
(\mathrm{Kbps})\end{array}$ \\
\hline 1 & 10 & 22.8 \\
2 & 12 & 34.3 \\
3 & 16 & 41.25 \\
4 & 19 & 51.6 \\
5 & 23 & 57.35 \\
6 & 28 & 69.2 \\
\hline
\end{tabular}


Table 2. Performance Comparison of Link-Adaptation Methods.

\begin{tabular}{|c|c|c|c|c|c|c|c|c|}
\hline \multirow{2}{*}{ Cases } & \multicolumn{6}{|c|}{ PER for Modulation Levels } & \multirow{2}{*}{$\begin{array}{c}\text { Overall } \\
\text { PER }\end{array}$} & \multirow{2}{*}{$\begin{array}{c}\text { Throughput } \\
\text { (Kbps) }\end{array}$} \\
\hline & 1 & 2 & 3 & 4 & 5 & 6 & & \\
\hline \multicolumn{9}{|c|}{ SINR-Based Adaptation Method } \\
\hline A) Without PC & 0.61 & 0.061 & 0.092 & 0.10 & 0.12 & 0.10 & 0.17 & 44.15 \\
\hline $\begin{array}{l}\text { B) Kalman PC } \\
\left(P_{R}=2 \%\right)\end{array}$ & 0.59 & 0.067 & 0.098 & 0.10 & 0.12 & 0.11 & 0.16 & 45.67 \\
\hline $\begin{array}{l}\text { C) Kalman PC } \\
\left(P_{R}=5 \%\right)\end{array}$ & 0.52 & 0.050 & 0.076 & 0.073 & 0.090 & 0.12 & 0.11 & 46.87 \\
\hline $\begin{array}{l}\text { D) Kalman PC } \\
\qquad\left(P_{R}=10 \%\right)\end{array}$ & 0.13 & 0.076 & 0.088 & 0.090 & 0.093 & 0.083 & 0.093 & 39.19 \\
\hline \multicolumn{9}{|c|}{ Terminal-Grouping Method for Adaptation } \\
\hline $\begin{array}{l}\text { E) Without PC } \\
\left(P_{R}=2 \%\right)\end{array}$ & 0.14 & 0.012 & 0.010 & 0.007 & 0.018 & 0.025 & 0.10 & 26.88 \\
\hline $\begin{array}{l}\text { F) Without PC } \\
\left(P_{R}=5 \%\right)\end{array}$ & 0.15 & 0.026 & 0.026 & 0.011 & 0.017 & 0.060 & 0.10 & 26.88 \\
\hline $\begin{array}{l}\text { G) Without PC } \\
\left(P_{R}=10 \%\right)\end{array}$ & 0.19 & 0.045 & 0.060 & 0.052 & 0.026 & 0.060 & 0.11 & 33.34 \\
\hline $\begin{array}{l}\text { H) Kalman PC } \\
\left(P_{R}=2 \%\right)\end{array}$ & 0.051 & 0.027 & 0.023 & 0.022 & 0.022 & 0.018 & 0.027 & 35.60 \\
\hline $\begin{array}{l}\text { I) Kalman PC } \\
\left(P_{R}=5 \%\right)\end{array}$ & 0.061 & 0.052 & 0.055 & 0.050 & 0.055 & 0.041 & 0.053 & 38.74 \\
\hline $\begin{array}{l}\text { J) Kalman PC } \\
\qquad\left(P_{R}=10 \%\right)\end{array}$ & 0.12 & 0.097 & 0.10 & 0.10 & 0.10 & 0.075 & 0.099 & 38.79 \\
\hline $\begin{array}{l}\text { K) Method D } \\
\text { in [LW99] }\end{array}$ & 0.052 & 0.023 & 0.031 & 0.045 & 0.045 & 0.028 & 0.029 & 32.7 \\
\hline
\end{tabular}


Table 3. Probability of Transmission at Various Modulation Levels for the Terminal-Grouping Method with 100\% Traffic Load.

\begin{tabular}{|c|l|l|l|l|l|l|l|}
\hline \multirow{2}{*}{ Terminal Group } & \multirow{2}{*}{ Target PER } & \multicolumn{7}{|c|}{ Modulation Level } \\
\cline { 3 - 7 } & & 1 & 2 & 3 & 4 & 5 & 6 \\
\hline \hline 1 & 0.05 & 1.0 & 0.0 & 0.0 & 0.0 & 0.0 & 0.0 \\
1 & 0.10 & 1.0 & 0.0 & 0.0 & 0.0 & 0.0 & 0.0 \\
1 & 0.15 & 1.0 & 0.0 & 0.0 & 0.0 & 0.0 & 0.0 \\
\hline 2 & 0.05 & 0.834 & 0.166 & 0.0 & 0.0 & 0.0 & 0.0 \\
2 & 0.10 & 0.646 & 0.354 & 0.0 & 0.0 & 0.0 & 0.0 \\
2 & 0.15 & 0.367 & 0.550 & 0.083 & 0.0 & 0.0 & 0.0 \\
\hline 3 & 0.05 & 0.180 & 0.603 & 0.218 & 0.0 & 0.0 & 0.0 \\
3 & 0.10 & 0.065 & 0.456 & 0.479 & 0.0 & 0.0 & 0.0 \\
3 & 0.15 & 0.033 & 0.423 & 0.544 & 0.0 & 0.0 & 0.0 \\
\hline 4 & 0.05 & 0.017 & 0.257 & 0.548 & 0.178 & 0.0 & 0.0 \\
4 & 0.10 & 0.0 & 0.103 & 0.542 & 0.338 & 0.017 & 0.0 \\
4 & 0.15 & 0.017 & 0.069 & 0.399 & 0.454 & 0.062 & 0.0 \\
\hline 5 & 0.05 & 0.0 & 0.0 & 0.033 & 0.562 & 0.372 & 0.033 \\
5 & 0.10 & 0.0 & 0.0 & 0.0 & 0.215 & 0.652 & 0.133 \\
5 & 0.15 & 0.0 & 0.0 & 0.0 & 0.149 & 0.600 & 0.251 \\
\hline 6 & 0.05 & 0.0 & 0.0 & 0.0 & 0.0 & 0.017 & 0.983 \\
\hline 5 & 0.10 & 0.0 & 0.0 & 0.0 & 0.0 & 0.0 & 1.0 \\
\hline 5 & 0.0 & 0.0 & 0.0 & 0.0 & 0.0 & 1.0 \\
\hline
\end{tabular}


Table 4. PER Performance of the Terminal-Grouping Method.

\begin{tabular}{|c|c|c|c|}
\hline Traffic Load $(\%)$ & Target PER & Measured PER $(L=10)$ & Measured PER $(L=5)$ \\
\hline \hline 100 & 0.02 & 0.027 & 0.028 \\
100 & 0.05 & 0.053 & 0.053 \\
100 & 0.10 & 0.099 & 0.099 \\
100 & 0.15 & 0.144 & 0.145 \\
100 & 0.20 & 0.188 & 0.191 \\
\hline 80 & 0.02 & 0.023 & 0.021 \\
80 & 0.05 & 0.050 & 0.049 \\
80 & 0.10 & 0.094 & 0.095 \\
80 & 0.15 & 0.136 & 0.141 \\
80 & 0.20 & 0.176 & 0.184 \\
\hline 60 & 0.02 & 0.023 & 0.022 \\
60 & 0.05 & 0.052 & 0.051 \\
60 & 0.10 & 0.097 & 0.097 \\
60 & 0.15 & 0.138 & 0.142 \\
60 & 0.20 & 0.171 & 0.183 \\
\hline 40 & 0.02 & 0.023 & 0.022 \\
40 & 0.05 & 0.050 & 0.051 \\
40 & 0.10 & 0.093 & 0.096 \\
40 & 0.15 & 0.128 & 0.139 \\
\hline & 0.20 & 0.161 & 0.179 \\
\hline
\end{tabular}


Fig.4. Throughput Performance of the Terminal-Grouping Method for $L=10$

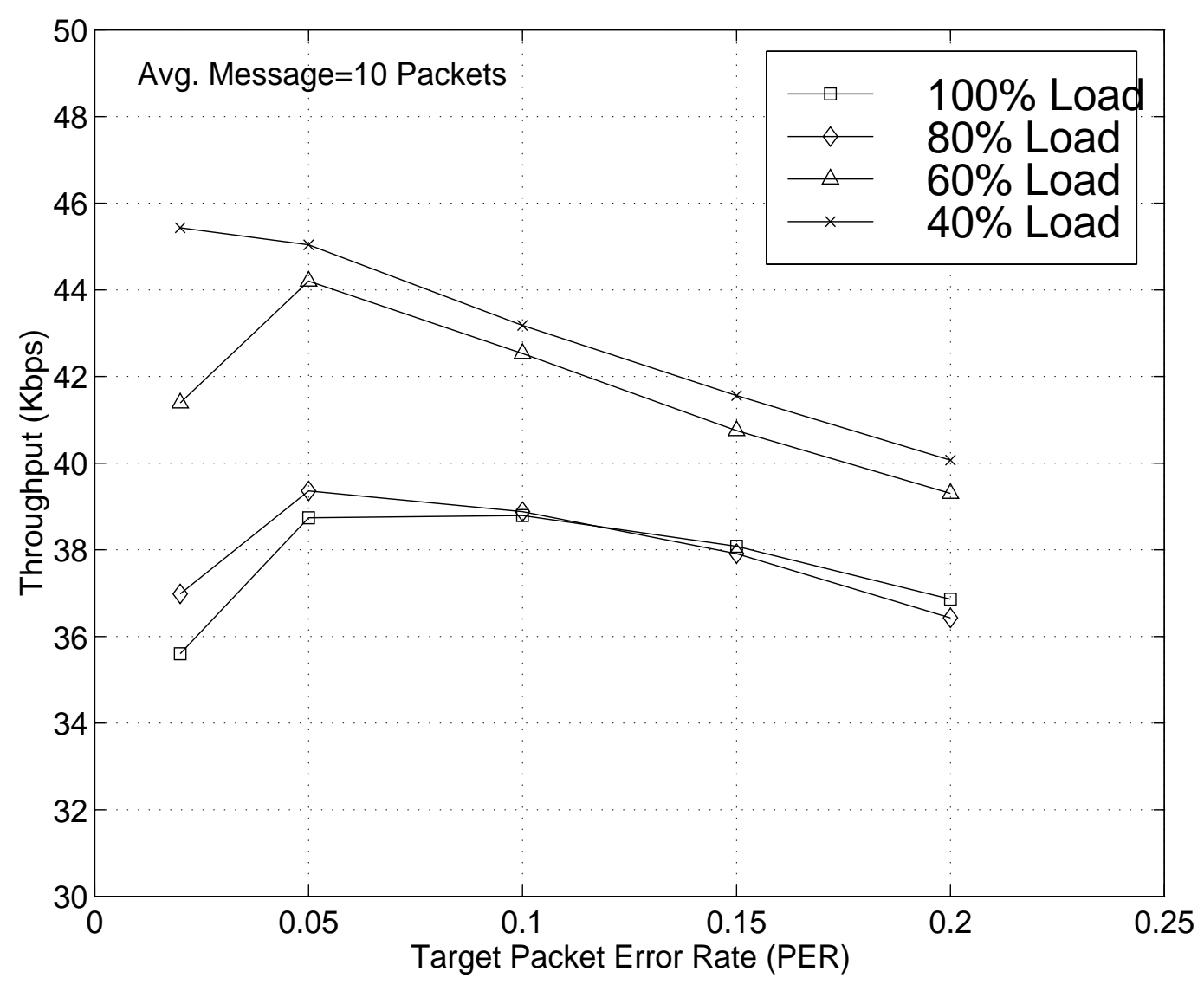


Fig.5. Throughput Performance of the Terminal-Grouping Method for $L=5$

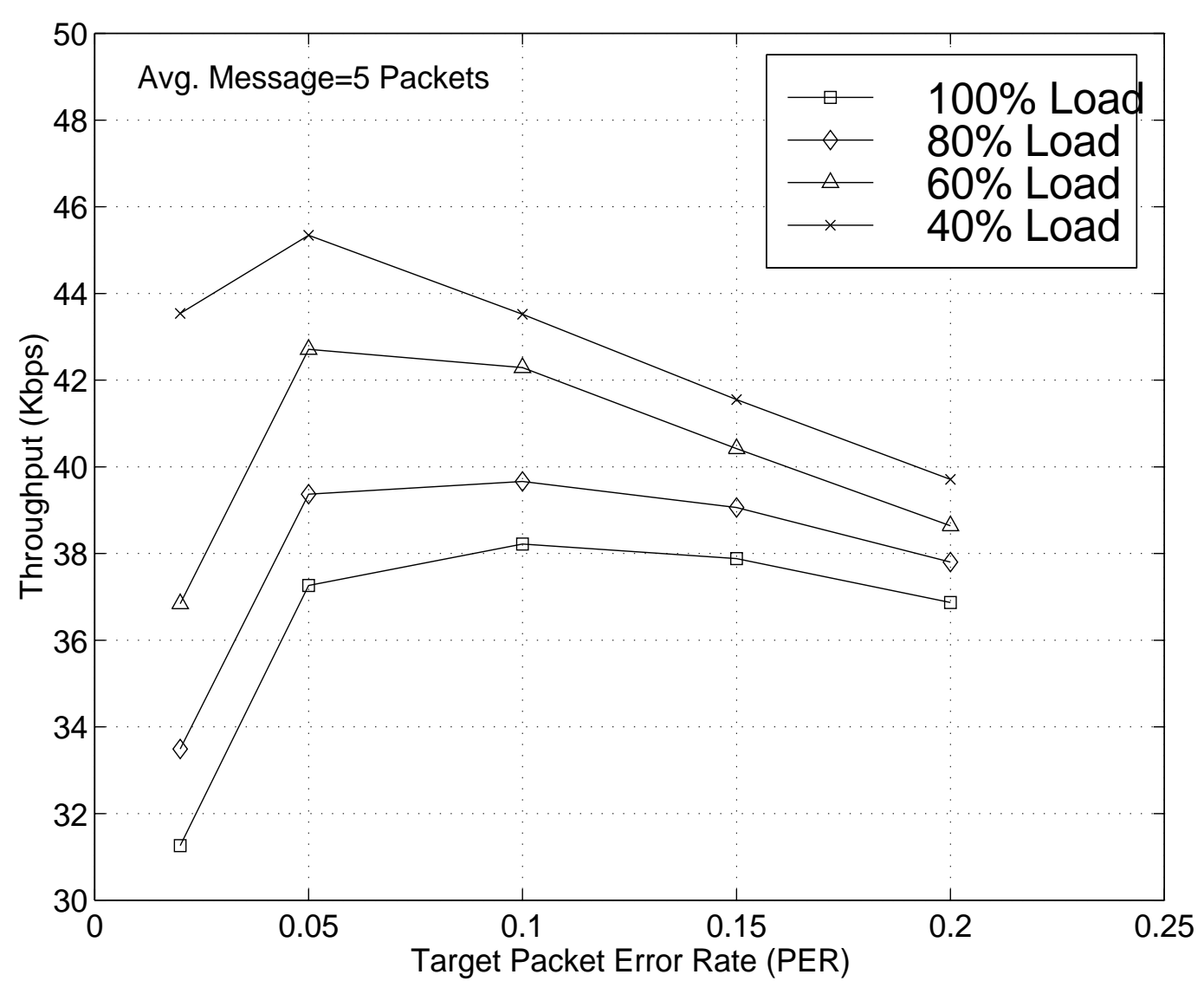

